\title{
Non-coding RNA Era is Dawning
}

\author{
Xue-feng Liu, Mary Hummel, and Michael Abecassis* \\ Northwestern University Feinberg School of Medicine, Comprehensive Transplant Center, Chicago, Illinois, 60611
}

Received: December 30, 2013; Accepted: January 13, 2014; Published: January 28, 2014

"Corresponding author: Michael Abecassis, Comprehensive Transplant Center, Northwestern University Feinberg School of Medicine, NMH/Arkes Family Pavilion Suite 1900, 676 N Saint Clair, Chicago IL 60611, USA, Tel: 312-695-8900; E-mail: mabecass@nmh.org

\begin{abstract}
Vast majority of transcripts in eukaryotic organisms are noncoding RNAs (ncRNAs). Except for traditional non-coding transcripts ribosomal RNA (rRNA) and transfer RNA (tRNA), both of which play essential roles in protein translation, most of ncRNAs haven't been functionally characterized and appreciated. Accumulating evidence, however, suggests that ncRNAs take part in many biological processes, and abnormal expression and/or dysfunction of them may result in a variety of diseases in human, such as cancers, neurodegenerative diseases, cardiovascular diseases and genetic diseases. The current understanding and future potential of ncRNAs in regulation of chromatin dynamics and gene expression, and implication to diseases are briefly outlined here.
\end{abstract}

\section{Introduction}

Up to $90 \%$ of eukaryotic genomes are transcribed. Only $2 \%$ of these transcripts encode proteins, while remaining $98 \%$ are ncRNAs [1,2]. ncRNAs can be categorized into infrastructural ncRNAs and regulatory ncRNAs. Constitutively expressed infrastructural ncRNAs include ribosomal RNAs (rRNAs), transfer RNAs (tRNAs), small nuclear RNAs (snRNAs), and small nucleolar RNAs (snoRNAs). Based on their size, regulatory ncRNAs can be grossly classified into small (<200nt) ncRNAs encompassing small interfering RNAs (siRNAs), microRNAs (miRNAs), and Piwi-interacting RNAs (piRNAs), and long ( >200nt) non-coding RNAs (lncRNAs) [2-4].

Certain members of infrastructural ncRNAs have wellcharacterized functions. For instance, rRNAs and tRNAs are integral for translation to take place in the cells. The snRNAs are components of spliceosomes, which carry out the splicing processes essential for removing intron sequences during mRNA maturation. The snoRNAs guide chemical modifications of rRNAs, and snRNAs $[5,6]$.

In addition, it has been determined that siRNAs and miRNAs regulate gene expression on post-transcriptional level through RNA cleaving and translational inhibition respectively, whereas piRNAs silence transposable elements (TEs) or imprinted loci of the genome through DNA methylation and/or histone modifications transcriptionally, and mRNA degradation or translation inhibition post-transcriptionally [7-10]. In this commentary, recent evidence that ncRNAs contribute to the maintenance of chromosomal structure, regulation of gene expression, and development of diseases will be highlighted.

\section{ncRNAs and chromosomal structures}

Telomeres are heterochromatic structures located on the ends of chromosomes, and protect the chromosomal DNA from being damaged. The highly repetitive nature of telomere regions causes them to be problematic in replicating, and thereby progressively shortened after each replication cycle, which is in turn overcome by the reverse transcribing enzyme telomerase. Recent studies demonstrated that eukaryotic telomeric ends are transcribed into IncRNAs named Telomeric Repeat containing RNAs (TERRA), which facilitate formation of telomeric heterochromatin, and inhibit telomerase from improperly lengthening the chromosomal ends [4]. Insufficient TERRA expression is correlated to breast cancer [11]. In early developmental stages of mammalian females, one of the pair of $\mathrm{X}$ chromosomes is transcriptionally silenced and condensed into a Barr body, and thereby provides equivalence between male and female in terms of products of genes sitting on $\mathrm{X}$ chromosomes (dosage compensation). X-inactive-specific transcript (Xist) is an IncRNA (17kb in human) transcribed from the inactive $\mathrm{X}$ chromosome, coats the chromosome in cis, and recruits Polycomb Repressive Complex 2 (PRC2) conferring H3K27me3, leading to inactivation. Xist is positively regulated by a group of IncRNAs arising from a region called $\mathrm{X}$ inactivation center (Xic), and negatively regulated by an antisense RNA, Tsix $[12,13]$.

\section{ncRNAs in gene regulation}

Several lines of evidence suggest that lncRNAs may regulate protein-coding gene expression in post-transcriptional level and transcriptional level [14-17]. Post-transcriptional regulation could be accomplished through miRNA sponge action of IncRNAs, thereby miRNA effector complexes are titrated away from their mRNA targets. Consequently, the mRNAs are spared from repression and allowed to be translated $[14,15]$. IncRNAs might regulation transcription in cis or trans, and positively or negatively. IncRNAs act in cis when they affect genes situated in the chromosomes from which they are transcribed, while in trans when they affect genes located on different chromosomes. 
One prototypical example of IncRNAs that act in trans is 331 nucleotide 7SK IncRNA, which halts transcriptional elongation by hampering the P-TEFb elongation factor from phosphorylating the RNA polymerase II carboxy terminal domain (CTD) [18]. Hox anti-sense intergenic RNA (HOTAIR) emanates from HOXC locus on chromosome 12, acts in trans to repress transcription around HOXD locus on chromosome 2 in human cells via targeting lysine specific demethylase1(LSD1) and PCR2, which can remove methyl groups from H3K4me2 and add methyl groups to H3K27 respectively, to the same genomic region $[17,18]$.

As opposed to trans-acting IncRNAs, which depend on their RNA products to exert their influence, cis-acting lncRNAs may operate in two distinct fashions. While long-range (nonoverlapped genes) regulation might entail their RNA products, the short range (overlapped genes) regulation could be carried out through 'transcriptional interference'(TI) where mere the procedure of IncRNA transcription is sufficient $[12,17]$ HOXA transcript at the distal tip (HOTTIP) is an $\sim 3.7 \mathrm{~kb}$ ncRNA transcribed from one end of the HOXA locus in mammalian cells, guides MLL1 (mixed lineage leukemia1) and WDR5 (WDrepeat containing protein5) histone modifying complex to promoter region of flanking genes via long range looping, and thereby causes trimethylation of $\mathrm{H} 3 \mathrm{~K} 4$ of the promoters and transcriptional activation of these genes $[12,17,18]$. Insulinlike growth factor type-2 receptor (Igf2r) is an imprinted gene, and silenced in paternal alleles [1]. Airn, a lncRNA expressed on paternally inherited chromosomes, overlaps the promoter of Igf2r gene in an antisense orientation, hinders the occupancy of functional RNA Polymerase II on Igf2r promoter in the context of open chromatin, leading to silencing of Igf2r gene $[12,17]$.

\section{ncRNAs and diseases}

As with protein-coding genes, mutation of ncRNA genes or aberrant expression of ncRNAs can cause a variety kinds of diseases. In Huntington's disease, for instance, there is an imbalance of neural lncRNA repertoire in brain tissues. While taurine upregulated1(TUG1) and nuclear paraspeckle assembly transcripts (NEAT1) are upregulated, maternally expressed 3 (MEG3) is down regulated [19]. The SNP rs133049 in the IncRNA called antisense noncoding RNA in INK4 locus (ANRIL) is reported to associate with myocardial infarction and pharmacogenomic evaluation in hypercholesterolemia [20]. ANRIL has been shown to be excessively expressed in prostate cancer cells. So does HOTAIR in breast cancer [21-23]. It has been demonstrated that Xist is downregulated in female breast cancer, ovarian cancer and cervical cancer cell lines, and suppresses haematologic cancer in vivo in mice $[23,24]$. Obviously, ncRNAs have been implicated in various diseases, and have potential to serve as diagnostic markers or therapeutic targets.

\section{Conclusion}

In a nutshell, ncRNAs play crucial roles in all facets of biological processes in both cellular level and organism level. Abnormality of ncRNAs has been implicated in numerous kinds of diseases, and might serve as useful resources for the development of diagnostic and interventional pharmacology. It is conceivable that more ncRNAs will be characterized in coming years, and ncRNA era is dawning.

\section{References}

1. Knowling S, Morris KV (2011) Noncoding RNA and antisense RNA. Nature's trash or treasure? Biochimie 93: 1922-1927.

2. Kaikkonen MU, Lam MTY, Glass CK (2011) Non-coding RNAs as regulator of gene expression and epigenetics. Cardiovasc Res 90: 430440 .

3. Guan D, Zhang W, Zhang W, Liu G-H, Izpisua Belmonte JC (2013) Switching cell fate, ncRNAs coming to play. Cell Death Dis 4: e464.

4. Brown JD, Mitchell SE, O’Neill RJ (2012) Making a long story short: noncoding RNA and chromosome change. Heredity 108: 42-49.

5. MatticK JS, Makunin IV (2006) Non-coding RNA. Hum Mol Genet 15: R17-29.

6. Scott MS, Ono M (2011) From snoRNA to miRNA: Dual function regulatory non-coding RNAs. Biochemie 93: 1987-1992.

7. van Wolfswinkel JC, Ketting RF (2010) The role of small non-coding RNA in genome stability and chromatin organization. J Cell Sci 123: 1825-1839.

8. Peng JC, Lin H (2013) Beyond transposons: the epigenetic and somatic functions of the Piwi-piRNA mechanism. Curr Opin Cell Biol 25: 190194.

9. Aalto AP, Pasquinelli AE (2012) Small non-coding RNAs mount a silent revolution in gene expression. Curr Opin Cell Biol 24: 333-340.

10. Ishizu H, Siomi H, Siomi MC (2012) Biology of PIWI-interacting RNAs: new insight into biogenesis and function inside and outside of germlines. Genes Dev 26: 2361-2373.

11. Cheetham SW, Gruhl F, Mattick JS, Dinger ME (2013) Long noncoding RNAs and genetics of cancer. Br J Cancer 108: 2419-2425.

12. Pauler FM, Barlow DP, Hudson QJ (2012) Mechanisms of long range silencing by imprinted macro non-coding RNAs. Curr Opin Genet Dev. 22: 283-289.

13. Caley DP, Pink RC, Trujillano D (2010) Long noncoding RNAs, chromatin, and development. ScientificWorldJournal 10: 90-102.

14. Yoon JH, Abdelmohsen K, Gorospe M (2013) Posttranscriptional gene regulation by long noncoding RNA. J Mol Biol 425: 3723-3730.

15. Cesana M, Cacchiarelli D, Legnini I, Santini T, Sthandier O, et al. (2011) A long noncoding RNA controls muscle differentiation by functioning as a competing endogenous RNA. Cell 147: 358-369.

16. Hu W, Alvarez-Domingues JR, Lodish HF (2012) Regulation of mammalian cell differentiation by long non-coding RNAs. EMBO reports 13: 971-983.

17. Kornienko AE, Guenzl PM, Barlow DP, Pauler FM (2013) Gene regulation by the act of long non-coding RNA transcription. BMC Biol 11: 59 .

18. Kugel JK, Goodrich JA (2012) Non-coding RNAs: Key regulators of mammalian transcription. Trends Biochem Sci 37: 144-151.

19. Tang JY, Lee JC, Chang YT, Hou MF, Huang HW, et al. (2013) Long noncoding RNAs-related diseases, cancer, and drugs. Scientific WorldJournal 2013: 943539.

20. Ahmed W, Ali IS, Riaz M, Younas A, Sadeque A, et al. (2013) Association of ANRIL polymorphism (rs133049: C > G) with myocardial infarction 
and its pharmacogenomic role in hypercholesterolemia. Gene 515: 416-420.

21. Kotake Y, Nakagawa K, Suzuki S, Liu N, Kitagawa M, et al. (2011) Long non-coding RNA ANRIL is required for the PRC2 recruitment to and silencing of p15 (INK4B) tumor suppressor gene. Oncogene 30: 19561962.

22. Pasmant E, Sabbagh A, Vidaud M, Bieche I (2011) ANRIL, a long noncoding RNA, is an unexpected hotspot in GWAS. FASEB J 25: 444448.
23. Gupta RA, Shah N, Wang KC, Kim J,. Horlings HM, et al. (2010) Long non-coding RNA HOTAIR reprograms chromatin state to promote cancer metastasis. Nature 464: 1071-1076.

24. Kawahami T, Zhang C, Taniguchi T, Kim CJ, Okada Y, et al. (2004) Characterization of loss of inactive $\mathrm{X}$ in Kinefelter syndrome and female-derived cancers. Oncogene 23: 6163-6169. 